LAWRENCE LIVERMORE N A T IO N A L LABORATORY
01-ERD-111 - The Development of Synthetic High Affinity Ligands

J. Perkins, R. Balhorn, M. Cosman, F. Lightstone, L. Zeller

February 17, 2004 
This document was prepared as an account of work sponsored by an agency of the United States Government. Neither the United States Government nor the University of California nor any of their employees, makes any warranty, express or implied, or assumes any legal liability or responsibility for the accuracy, completeness, or usefulness of any information, apparatus, product, or process disclosed, or represents that its use would not infringe privately owned rights. Reference herein to any specific commercial product, process, or service by trade name, trademark, manufacturer, or otherwise, does not necessarily constitute or imply its endorsement, recommendation, or favoring by the United States Government or the University of California. The views and opinions of authors expressed herein do not necessarily state or reflect those of the United States Government or the University of California, and shall not be used for advertising or product endorsement purposes.

This work was performed under the auspices of the U.S. Department of Energy by University of California, Lawrence Livermore National Laboratory under Contract W-7405-Eng-48. 


\section{1-ERD-111 - The Development of Synthetic High Affinity Ligands}

Principal Investigator: Julie Perkins

Co-Investigators: Rod Balhorn, Monique Cosman, Felice Lightstone, Lori Zeller

Introduction. The aim of this project was to develop Synthetic High-Affinity Ligands (SHALs), which bind with high affinity and specificity to proteins of interest for national security and cancer therapy applications. The aim of producing synthetic ligands for sensory devices as an alternative to antibodybased detection assays and therapeutic agents is to overcome the drawbacks associated with antibodybased in next-generation sensors and systems. The focus area of the project was the chemical synthesis of the SHALs.

The project concentrated on two different protein targets. (a) The $\mathrm{C}$ fragment of tetanus and botulinum toxin, potential biowarfare agents. A SHAL for tetanus or botulinum toxin would be incorporated into a sensory device for the toxins. (b) HLA-DR10, a protein found in high abundance on the surface of NonHodgkins Lymphoma. A SHAL specific to a tumor marker, labeled with a radionuclide, would enable the targeted delivery of radiation therapy to metastatic disease.

The technical approach used to develop a SHAL for each protein target will be described in more detail below. However, in general, the development of a SHAL requires a combination of computational modeling techniques, modern nuclear magnetic resonance spectroscopy (NMR) and synthetic chemistry.

Results and Discussion - Tetanus and Botulinum toxin. The X-ray crystal structures of the C fragment of tetanus and botulinum toxin have previously been determined. The structure of the $\mathrm{C}$ fragment of tetanus and botulinum toxin are so similar that a SHAL for one is likely to bind the other. Therefore, the $\mathrm{C}$ fragment of tetanus toxin was generally used in the ligand development experiments. Using the crystal structure of tetanus toxin, computational modeling techniques were used to identify unique pockets on the surface of the protein. Subsequently, the Available Chemicals Directory (ACD) (MDL Information Systems, CA) was computationally screened for molecules that bind into the unique pockets. The resultant short-list of molecules, termed low affinity ligands, were subsequently experimentally tested for their affinity for tetanus toxin using mass spectrometry and a liposome assay. The full details of these experiments can be found in the publication: Lightstone et al, Chem. Res. Toxicol. 2000, 13, 356-362. In addition, NMR spectroscopy was used to experimentally screen low affinity ligands for their solution-phase affinity for tetanus toxin. The detailed results of these experiments are described in the publication: Cosman et al, Chem. Res. Toxicol. 2002, 15, 1218-1228.

Sets of two low affinity ligands from the shortlist, one for each pocket, were selected for development as SHALs, initially doxorubicin (Pocket 1) and the peptide sequences YEW and SQNYPIV (Pockets 2). 
External collaborators have also shown that doxorubicin and YEW bind to botulinum toxin in different, but closely located pockets, using X-ray crystallography. In order to create a ligand with higher affinity for a protein, bidentate ligands were constructed. The bidentate or the multidentate effect is a well know phenomenon in nature. The linking of two low affinity ligands using a optimal linker length and type can result in a high affinity ligand. The affinity of the new ligand can be several orders of magnitude higher than the sum of the affinity of the low affinity ligands.

As a starting point, lysine was used as a linking system, as the many commercially available derivatives of lysine make synthesis of amide-based ligands more simple. In addition, a short polyethylene glycol linker was used as a spacer between to two low affinity ligands, as varying the length of the linker in a SHAL is an important step in bidentate ligand development to achieve optimum binding.

Initially, doxorubicin and biotin (another low affinity ligand identified in the screening process) were linked together with two different length linkers. One of the resulting SHALs was shown to higher affinity for tetanus toxin than doxorubicin itself. However, biotin is not ideal as a main constituent of a SHAL, and therefore, synthetic work in constructing a SHAL with a biotin tag (for easy of subsequent screening against the toxin) with either doxorubicin or SQNYPIV and YEW was initiated. The initial efforts to synthesize biotin-labeled SHALs with SQNYPIV were unsuccessful, and a two step approach was required. However, the synthesis of SHALs for tetanus toxin was not completed within the timeframe of this project, and therefore no further results in this area were obtained. This is mainly a consequence of the success, and therefore concentrating efforts, in developing a SHAL for HLA-DR10, which is described in more detail below.

Results and Discussion - HLA-DR10. The crystal structure of HLA-DR10 is not available. Therefore a computational model was constructed. Subsequently, unique pockets on the surface of HLA-DR10 were identified. In a manner similar to that for tetanus toxin, the ACD was screened for low affinity ligands.

Sets of two low affinity ligands, one for each site, from the shortlist were selected for development as SHALs. The low affinity ligands leu-enkephalin and deoxycholate were the first set selected for development. Again, a lysine backbone and short polyethylene glycol linkers were used to sythesize biotin-labeled SHALs for HLA-DR10. Solid-phase chemistry was used to construct three SHALs containing leu-enkephalin and deoxycholate with zero, one and two polyethylene glycol linkers between the low affinity ligands. In addition, control molecules, biotin-labeled leu-enkephalin and deoxycholate, were also synthesized. The testing of the SHALs was carried out using surface plasmon resonance experiments on streptavidin chips and NMR experiments. The SHAL with one polyethylene glycol linker was shown to have high affinity for the HLA-DR10. The binding affinity of the SHAL is in a similar 
range to that of the natural antibody to HLA-DR10, Lym-1. External collaborators at UC Davis Cancer Center have tested this SHAL against fixed tumor tissue and the SHAL continues to show high affinity for the target HLA-DR10. Control experiments against normal tissue other cancer tissues are also currently underway with SHAL synthesized as part of this project. Following the success with the biotinlabeled SHAL, a SHAL containing a chelating agent for a radionuclide was synthesized. The main "binding" part of the SHAL was exactly the same as for the biotin-labeled SHAL, however, the biotin was replaced with a well know chelating agent for radionuclides, which already used in other cancer therapeutics. Initial efforts to label the chelator-tagged SHAL with a radionuclide were unsuccessful. However, this was a consequence of many different factors, and these experiments are to be repeated by external collaborators.

Conclusions. The encouraging results obtained in this project for the development of SHALs for HLADR10 has enabled the researchers to pursue external funding in order to continue work in this area. Future experiments include the repeating of the radiolabeling the most successful SHAL to date, and subsequently testing its behavior both in vitro and in vivo. The results of these experiments will dictate the future direction of the development of SHALs as therapeutic agents against Non-Hodgkins Lymphoma. 